\title{
Error correction of transformed rectangular model of concave and convex MAGFETs with AC bias
}

\author{
G.-M. Sung and S.-I. Liu
}

\begin{abstract}
The authors derive and experimentally confirm the error correction factor, which is used to compensate for the induced current difference, according to the geometric parameters of concave and convex MAGFETs. The measurement results indicate that the highest absolute sensitivity $S_{A}$, the supply-current-related sensitivity $S_{R I}$ and the supply-voltage-related sensitivity $S_{R V}$, are $88.82 \mathrm{mV} / \mathrm{T}, 2121.74 \mathrm{~V} / \mathrm{A} . \mathrm{T}$ and $177.65 \mathrm{mV} /$ V.T, respectively, for concave MAGFETs and $S_{A}=261.34 \mathrm{mV} / \mathrm{T}, S_{R I}=6008.26 \mathrm{~V} / \mathrm{A} . \mathrm{T}$ and $S_{R V}=522.68 \mathrm{mV} / \mathrm{V}$.T for convex MAGFETs with $0.5 \mathrm{~V} \mathrm{AC}$ bias voltage, which is applied with high-frequency, $100 \mathrm{kHz}$, to decrease the $1 / f$ noise. The sensitivities of convex MAGFETs are greater than those of concave MAGFETs, and the transformed model needs to be built with a low aspect ratio $(L / W)$ and a high drain gap $(d)$. Given a consistent geometric correction factor, the convex MAGFET with geometric parameters, $L /$ $W=40 \mu \mathrm{m} / 80 \mu \mathrm{m}$ and $d=2 \mu \mathrm{m}$, is the best choice, and the concave MAGFET with $L / W=40 \mu \mathrm{m} /$ $40 \mu \mathrm{m}$ and $d=4 \mu \mathrm{m}$, is another good choice. Both a broadened drain gap and a small aspect ratio reduce the measured geometric correction factor $(G)$ by increasing the magnitude of the error correction factor.
\end{abstract}

\section{Introduction}

Several studies have proposed and experimentally verified complementary split-drain MAGFET pairs with circuits and numerical models to detect galvanomagnetic effects [1-3]. Although operating with sensitivities higher than those of conventional magnetic devices, the MAGFET suffers from the $1 / f$ noise problem and the offset of the ordinary MOSFET, which seriously limit its performance [4]. Accordingly, this work presents a novel solution by using AC bias modulation at the gate of the MAGFET. First, we increase the operating frequency $100 \mathrm{kHz}$ of the AC bias voltage to reduce the $1 / f$ noise. Secondly, the DC offset, which is generated by the DC bias voltage, must be calibrated to a minimum value before the AC bias voltage is added on. Measuring the difference in current induced by the applied DC magnetic induction is a vital task. The operating principle of $\mathrm{AC}$ bias modulation is assumed to depend on the giant magneto-impedance effect, GMI, which has been interpreted in classical electrodynamics as a change in the depth of penetration of the AC current that flows in the magnetic slab, caused by the applied DC magnetic field [5].

The shape of the MAGFET must be transformed into an equivalent rectangle to derive the induced current difference and satisfy the operating principle of the traditional Hall plate [6]. This transformation enables

\footnotetext{
C) IEE, 2004
}

IEE Proceedings online no. 20040255

doi:10.1049/ip-cds:20040255

Paper first received 14th March and in revised form 29th August 2003. Originally published online: 18th May 2004

G.-M. Sung is with the Department of Electrical Engineering, National Taipe University of Technology, 1, Sec. 3, Chung-Hsiao E. Rd. Taipei 106, Taiwan, Republic of China

S.-I. Liu is with the Department of Electrical Engineering and Graduate Institute of Electronic Engineering, National Taiwan University, 1, Sec. 4, Roose-Fu Rd. Taipei 106, Taiwan, Republic of China the proposed theoretical model to fit any magnetic devices. However, the change in device structure and the short-circuiting caused by the finite current and sensing contacts will in fact yield an inevitable error between the ideal and real devices [7]. For industrial applications, the geometric correction factor must be modified to eliminate unexpected errors. An error correction factor is introduced accordingly.

Figures 1 and 2 illustrate the proposed $n$-channel concave and convex MAGFETs with channel length $L$, channel width $W$, drain gap width $d$ and drain gap length $u$ (fixed at $2.8 \mu \mathrm{m})$. The MAGFETs were examined using a SPICE macro model and proven to perform electrically like a standard MOS transistor [8]. When a magnetic field $\boldsymbol{B}$ is applied perpendicular to the device surface, a Lorenz force pushes the charges or carriers to one side and thereby increases the drain current; a simultaneous reduction occurs in the counterpart device. Thus, the current difference in the MOSFET $\Delta I=I_{D 1}-I_{D 2}$, induced by DC magnetic induction, is measured to assess the magnetic effect [9]. After this current difference passes through a readout circuit (shown in Fig. 3), an output voltage $\Delta V_{\text {out }}=2 \times \Delta I \times R_{\text {out }}$, can be obtained with an output resistance of $R_{\text {out }}=10 \mathrm{k} \Omega$. The characteristics of an operational amplifier are constrained by a low offset and a high-frequency response $\left(f_{T}\right.$ above $\left.3 \mathrm{MHz}\right)$ to prevent undesired deviations and distortions [10]. The geometric correction factor is rewritten as

$$
G=\frac{V_{H}}{V_{\text {Hideal }}}=\frac{\Delta V_{\text {out }}}{2 R_{\text {out }} \times \Delta I_{\text {ideal }}}
$$

where $\Delta I_{\text {ideal }}$ represents the value calculated according to the operating principle. Moreover, the arrayed and symmetrical layouts are also considered to enhance the output voltage and inhibit the unwanted noise [11]. Four magnetic devices were used in combination to increase the current difference, and the measured results imply that this array is more sensitive than a single device. 


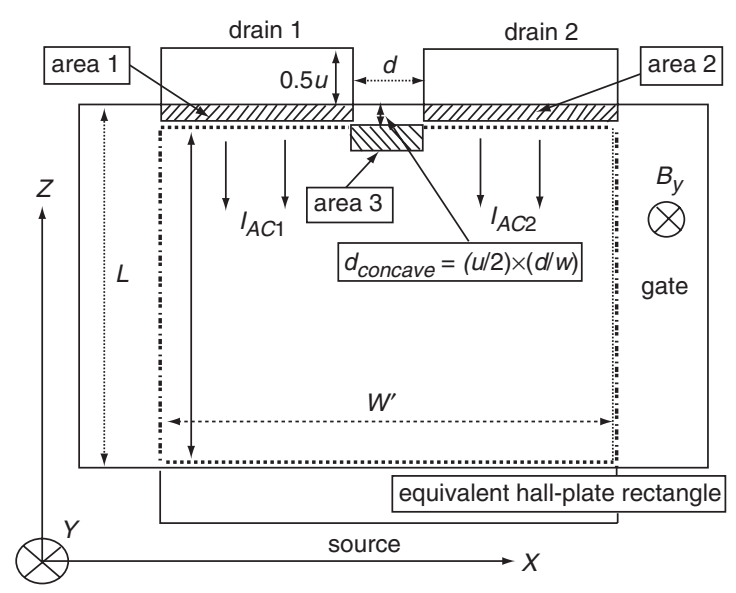

Fig. 1 Layout and equivalent rectangular model of an n-channel concave MAGFET

Geometric parameters $L, W, d$ and $u$ represent channel length, channel width, drain gap width and drain gap length, respectively; $L^{\prime}$ and $W^{\prime}$ are the equivalent length and width; $I_{A C 1}$ and $I_{A C 2}$ are the AC drain currents (bias current) of drains 1 and 2; areas 1 and 2 are defined to fill area 3. For definition of $L^{\prime}$, see (5)

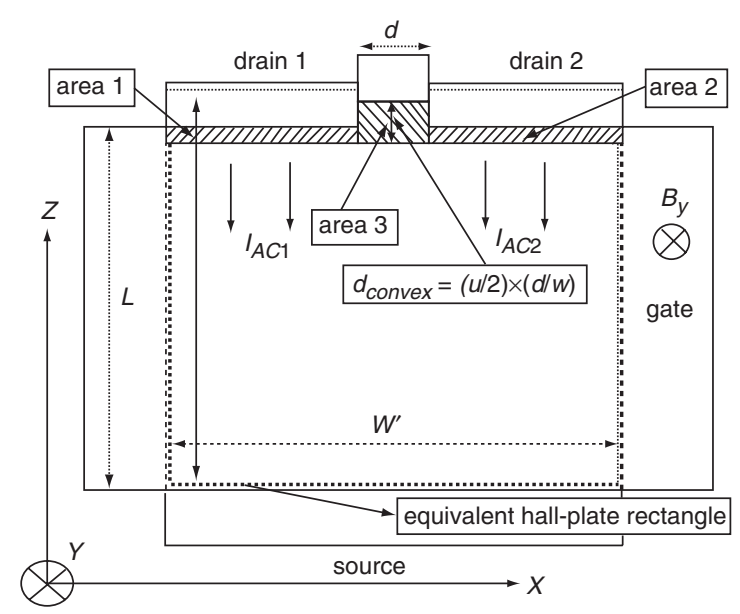

Fig. 2 Layout and equivalent rectangular model of an n-channel convex MAGFET with geometric parameters as defined in Fig. 1

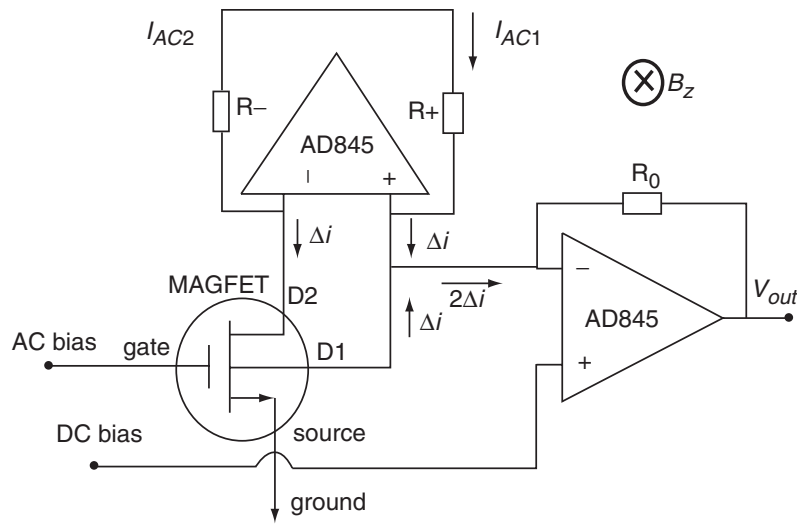

Fig. 3 Readout circuit of a MAGFET used to convert the induced $A C$ current difference $\Delta i$ into the $A C$ output voltage $\Delta V_{\text {out }}$ by passing through the output resistance $R_{\text {out }}$

$V_{\text {out }}=2 \Delta i \times R_{\text {out }}$

$I_{A C}=I_{A C 1}+I_{A C 2}$

\section{Operating principles}

\subsection{Equivalent rectangle model}

As Kluge et al. stated [6], concave and convex MAGFETs must first be transformed into two Hall-plate rectangular models to satisfy the general principles of operation. In the equivalent rectangular models, all the drain gaps are zero. The 'equivalent area' concept is used to determine the indentation or protrusion distances $d_{\text {concave }}$ and $d_{\text {convex }}$. For a concave MAGFET, areas 1 and 2 are defined to fill the depressed area 3 in Fig. 1. Thus, the indentation distance $d_{\text {concave }}$ is determined by

$$
d_{\text {concave }}=\frac{u}{2 W} \times d
$$

The transformed channel length $L^{\prime}$, can be calculated from the potential distribution $V(z)$, which is defined as $[6,12]$ :

$$
V(z)=V_{D S}\left(\frac{z}{L}\right)^{1 /\left(1-k \frac{d}{W}\right)}
$$

where $k$ is the drain-gap degradation factor, $V_{D S}$ is the voltage drop from the drain to the source and $z$ is the coordinate in Fig. 1. Differentiating the potential $V(z)$ yields the electric field $E$ at the effective position $z=L-d_{\text {concave }}$.

$$
\left|E\left(z=L-\frac{u d}{2 W}\right)\right|=\frac{L\left(1-k \frac{d}{W}\right)}{\left(1-\frac{1}{2} \times \frac{u}{L} \times \frac{d}{W}\right)^{k d /(W-k d)}}
$$

Given a homogeneous electric field $\left(E=V_{D S} / L^{\prime}=\right.$ constant), the equivalent channel length is

$$
\begin{aligned}
L^{\prime} & =\frac{L\left(1-k \frac{d}{W}\right)}{\left(1-\frac{1}{2} \times \frac{u}{L} \times \frac{d}{W}\right)^{k d /(W-k d)}} \\
& \cong L\left(1-k \frac{d}{W}\right)\left(1+\frac{k}{2} \times \frac{u}{L} \times \frac{d}{W} \times \frac{d}{W-k d}\right)
\end{aligned}
$$

Clearly, an increase in the drain gap $d$ of the original concave MAGFET will reduce the equivalent channel length in this equivalent concave model.

For a convex MAGFET (shown in Fig. 2), a similar derivation is performed, assuming the potential is

$$
V(z)=V_{D S}(z / L)^{1 /\left(1+k \frac{d}{W}\right)}
$$

The protrusion distance $d_{\text {convex }}$ and the equivalent channel length $L^{\prime}$ are obtained as

$$
d_{\text {convex }}=\frac{u}{2 W} \times d
$$

and

$$
L^{\prime} \cong L\left(1+k \frac{d}{W}\right)\left(1+\frac{k}{2} \times \frac{u}{L} \times \frac{d}{W} \times \frac{d}{W+k d}\right)
$$

Notably, the channel width $W$ is not degraded by the drain gap, so that the equivalent channel width $W^{\prime}$ equals the initial channel width $W$. That is, $W^{\prime}=W$.

\subsection{Current difference and current correction factor}

Assuming that a small magnetic field $(B \ll 1 \mathrm{~T})$, perpendicular to the chip surface, is applied in the $y$ direction, and neglecting the carrier generation and recombination, the current difference $\Delta I$ at the drains $\mathrm{D} 1$ and $\mathrm{D} 2$ can be derived from the electron current density $\boldsymbol{J}_{n}$, which satisfies the following equation from Baltes and Popovic [13].

$$
\boldsymbol{J}_{n}(\boldsymbol{B})=\sigma_{n B}\left\lfloor\boldsymbol{E}+\mu_{n}^{*}(\boldsymbol{E} \times \boldsymbol{B})\right\rfloor
$$

where $\sigma_{n B}=\sigma_{n}\left[1+\left(\mu_{n}^{*}\right)^{2}(\boldsymbol{B} \bullet \boldsymbol{B})\right]^{-1}$ and $\mu_{n}^{*}$ represents the Hall mobility of electrons, $\mu_{n}^{*}=r_{n} \mu_{n}$, and the scattering 
factor $r_{n}=\left\langle\tau_{n}^{2}\right\rangle /\left\langle\tau_{n}\right\rangle^{2}$, where $\tau_{n}$ is the free time between collisions. Given $\boldsymbol{B}=\left(0, B_{y}, 0\right), \quad \boldsymbol{E}=\left(E_{x}, 0, E_{z}\right)$ and $\boldsymbol{J}_{n}(\boldsymbol{B})=\left(J_{n x}, 0, J_{n z}\right),(8)$ can be divided into two equations:

$$
\begin{gathered}
J_{n x}\left(B_{y}\right)=\sigma_{n B}\left[E_{x}-\mu_{n}^{*} E_{z} B_{y}\right] \\
J_{n z}\left(B_{y}\right)=\sigma_{n B}\left[E_{z}+\mu_{n}^{*} E_{x} B_{y}\right] \cong \sigma_{n B} E_{z}
\end{gathered}
$$

where $E_{x}$ is induced by the Lorenz force in the $x$-direction and is small. Then, substituting the equation $E_{x}=-G_{A C} \mu_{n} E_{z} B_{y}$, into (9) and applying an AC geometric correction factor $G_{A C}$, the electron current density $J_{n x}$ is

$$
\begin{aligned}
J_{n x}\left(B_{y}\right) & =-\sigma_{n B} E_{z} B_{y} \mu_{n}\left[G_{A C}+r_{n}\right] \\
& \cong-J_{n z} \mu_{n}\left[G_{A C}+r_{n}\right] B_{y}
\end{aligned}
$$

Accordingly, the current difference of the concave MAGFET can be determined by integrating (11) over the equivalent rectangular model in the $\mathrm{z}$-direction from $z=0$ to $z=L-(u / 2) \times(d / W)$. Then,

$$
\begin{aligned}
& \int_{0}^{L-\frac{u}{2} \times \frac{d}{W}} \int_{0}^{W} \int_{0}^{t} J_{n x}\left(B_{y}\right) d y d x d z \\
& =\int_{0}^{L-\frac{u}{2} \times \frac{d}{W}} \int_{0}^{W} \int_{0}^{t}\left(-J_{n z} \mu_{n}\left[G_{A C}+r_{n}\right] B_{y}\right) d y d x d z
\end{aligned}
$$

The current differences in the $x$ direction $\Delta I_{x}$ are obtained from

$$
\Delta I_{x} \times W=-\mu_{n} B_{y} I_{z} \int_{0}^{L-\frac{u}{2} \times \frac{d}{W}}\left[G_{A C}+r_{n}\right] d z
$$

where $t$ is the thickness of the MAGFET along the direction of the magnetic field, and

$$
\begin{aligned}
\Delta I_{x} & =\int_{0}^{L-\frac{u}{2} \times \frac{d}{W}} \int_{0}^{t} J_{n x}\left(B_{y}\right) d y d z \\
I_{z} & =\int_{0}^{W} \int_{0}^{t} J_{n z} d y d x
\end{aligned}
$$

An error correction factor $E_{\text {concave }}$ can be inserted into (13) to compensate for the current difference of the concave MAGFET $\Delta I_{\text {concave }}$.

$$
\Delta I_{\text {concave }}=-\mu_{n} B_{y} I_{z} \frac{1}{W} \int_{0}^{L-\frac{u}{2} \times \frac{d}{W}}\left[E_{\text {concave }} G_{A C}+r_{n}\right] d z
$$

and the current difference of the convex MAGFET $\Delta I_{\text {convex }}$ is

$$
\Delta I_{\text {convex }}=-\mu_{n} B_{y} I_{z} \frac{1}{W} \int_{0}^{L+\frac{u}{2} \times \frac{d}{W}}\left[E_{\text {convex }} G_{A C}+r_{n}\right] d z
$$

where $E_{\text {convex }}$ is the error correction factor that compensates for the current difference in the convex MAGFET. The error correction factors, $E_{\text {concave }}$ and $E_{\text {convex }}$, can be easily corrected by replacing the known variables [12, 14]. However, determining the $\mathrm{AC}$ geometric correction factor is critical. In the following Section, it is derived from Laplace's equation.

\subsection{AC geometric correction factor $G_{A C}$}

The effect of an AC driving bias current can be determined from Laplace's equation:

$$
\nabla^{2} V(x, z)=\frac{\partial^{2} V}{\partial x^{2}}+\frac{\partial^{2} V}{\partial z^{2}}=0 \quad \text { (in two dimensions) }
$$

where $V(x, z)$ is the voltage distribution with respect to the $x$ axis and the $z$ axis. (See Fig. 4.) The following boundary conditions (BC) and initial conditions (IC) are assumed:

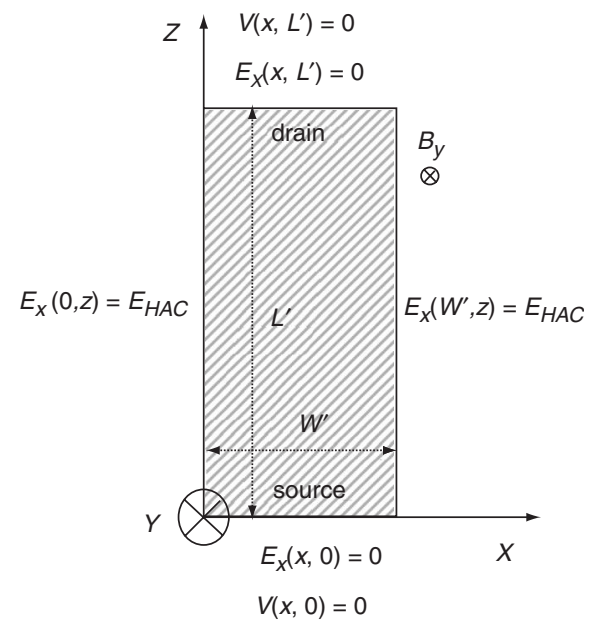

Fig. 4 Transformed Hall-plate rectangle with initial and boundary conditions

$E_{x}, V$ and $E_{H A C}$ are the electrical field along the x-axis, the bias voltage distribution and the Hall electrical field induced by the AC bias voltage, respectively

BC:

$$
\begin{aligned}
& E_{x}(x, 0)=E_{x}\left(x, L^{\prime}\right)=0 \\
& E_{x}(0, z)=E_{x}\left(W^{\prime}, z\right)=E_{H A C}
\end{aligned}
$$

IC:

$$
\begin{aligned}
& V(x, 0)=0 \\
& V\left(x, L^{\prime}\right)=V_{\text {bias }}
\end{aligned}
$$

in which $E_{x}, V_{\text {bias }}$ and $E_{H A C}$ are the electrical field in the $x$ direction, the AC bias voltage and the electrical field induced by the Hall effect of the AC bias voltage, respectively.

From the boundary and initial conditions (shown in the Appendix) [15],

$$
\begin{aligned}
V(x, z)= & \sum_{n=1}^{\infty}\left[\frac{2 E_{H A C} \cdot L^{\prime} \cdot\left[(-1)^{n}-1\right]}{(n \pi)^{2}} \cdot \frac{1}{\sinh \frac{n \pi}{L^{\prime}} W^{\prime}}\right] \\
& \cdot\left[\cosh \frac{n \pi}{L^{\prime}} x \cdot \sin \frac{n \pi}{L^{\prime}} z\right] \\
& +\sum_{n=1}^{\infty}\left[\frac{-2 L^{\prime} \cdot E_{H A C} \cdot\left[(-1)^{n}-1\right]}{(n \pi)^{2}} \cdot \frac{1}{\tanh \frac{n \pi}{L^{\prime}} W^{\prime}}\right] \\
& \cdot\left[\cosh \frac{n \pi}{L^{\prime}} x-\tanh \frac{n \pi}{L^{\prime}} W^{\prime} \cdot \sinh \frac{n \pi}{L^{\prime}} x\right] \cdot \sin \frac{n \pi}{L^{\prime}} z \\
& +f(z)
\end{aligned}
$$

where $f(z)$ is the voltage distribution, which is assumed linear with a sensing position $z$. That is, $f(z)=a z+b$. Substituting the initial values $V(x, 0)=0$ and $V\left(x, L^{\prime}\right)=V_{\text {bias }}$, into (16) yields $f(z)=\left(V_{\text {bias }} / L^{\prime}\right) z$. Thus,

$$
\begin{aligned}
V(x, z)= & \sum_{n=1}^{\infty} \frac{2 E_{H A C} \cdot L^{\prime} \cdot\left[(-1)^{n}-1\right]}{(n \pi)^{2}} \\
& \cdot\left[\frac{1-\cosh \frac{n \pi}{L^{\prime}} W^{\prime}}{\sinh \frac{n \pi}{L^{\prime}} W^{\prime}} \cdot \cosh \frac{n \pi}{L^{\prime}} x+\sinh \frac{n \pi}{L^{\prime}} x\right] \\
& \times\left[\sin \frac{n \pi}{L^{\prime}} z\right]+\frac{V_{\text {bias }}}{L^{\prime}} z
\end{aligned}
$$


The AC geometric correction factor $G_{\mathrm{AC}}$ is defined as the ratio of the induced Hall voltage of the real magnetic device to that of the ideal magnetic device. Then it is rewritten as

$$
\begin{aligned}
G_{A C}\left(L^{\prime} / W^{\prime}\right)= & \frac{\left.V(x, z)\right|_{x=W^{\prime}}-\left.V(x, z)\right|_{x=0}}{-E_{H A C} \cdot W^{\prime}} \\
= & \sum_{n=1}^{\infty} \frac{2 L^{\prime} \cdot\left[1-(-1)^{n}\right]}{W^{\prime} \cdot(n \pi)^{2}} \\
& \cdot\left[-\frac{\left(1-\cosh \frac{n \pi}{L^{\prime}} W^{\prime}\right)^{2}}{\sinh \frac{n \pi}{L^{\prime}} W^{\prime}}+\sinh \frac{n \pi}{L^{\prime}} W^{\prime}\right] \\
& \cdot \sin \frac{n \pi}{L^{\prime}} z
\end{aligned}
$$

When the sensing position is set at the centre of the equivalent rectangular model, such that $z=\frac{1}{2} L^{\prime}$, the $\mathrm{AC}$ geometric correction factor becomes

$$
\begin{aligned}
G_{A C}\left(L^{\prime} / W^{\prime}\right)= & \sum_{n=1}^{\infty} \frac{2 L^{\prime} \cdot\left[1-(-1)^{n}\right]}{W^{\prime} \cdot(n \pi)^{2}} \\
& \cdot\left[-\frac{\left(1-\cosh \frac{n \pi}{L^{\prime}} W^{\prime}\right)^{2}}{\sinh \frac{n \pi}{L^{\prime}} W^{\prime}}+\sinh \frac{n \pi}{L^{\prime}} W^{\prime}\right] \\
& \cdot \sin \frac{n \pi}{2}
\end{aligned}
$$

The central sensing position is clearly the best choice for the equivalent rectangular Hall plate, and the aspect ratio $\left(L^{\prime} / W^{\prime}\right)$ and the drain gap $(d)$ are the key parameters that govern the AC geometric correction factor. Figure 5 presents the simulated results for the geometric correction factor $G_{A C}$ with respect to the aspect ratio $L^{\prime} / W^{\prime}$. Notably, a sharp variation in the $\mathrm{AC}$ geometric correction factor occurs during the aspect ratio interval between zero and two. Another important parameter $d$ (drain gap) is of interest. Figure 6 plots the relationship between the geometric correction factor and drain gap. Simulation results indicate that the amplitude of function $G_{A C}$ slowly decreases as the drain gaps are increased stepwise. According to the literature [8] and with a view to obtaining an optimum output, the section of the drainage gap of $2-$ $6 \mu \mathrm{m}$, with a step of $2 \mu \mathrm{m}$, is considered. Although the simulation results show that the magnetic device with a high aspect ratio and a small drain gap may exhibit the optimum AC geometric correction factor $(\approx 1)$, the nonlinearity, which is induced by the coupling drain current, and the low

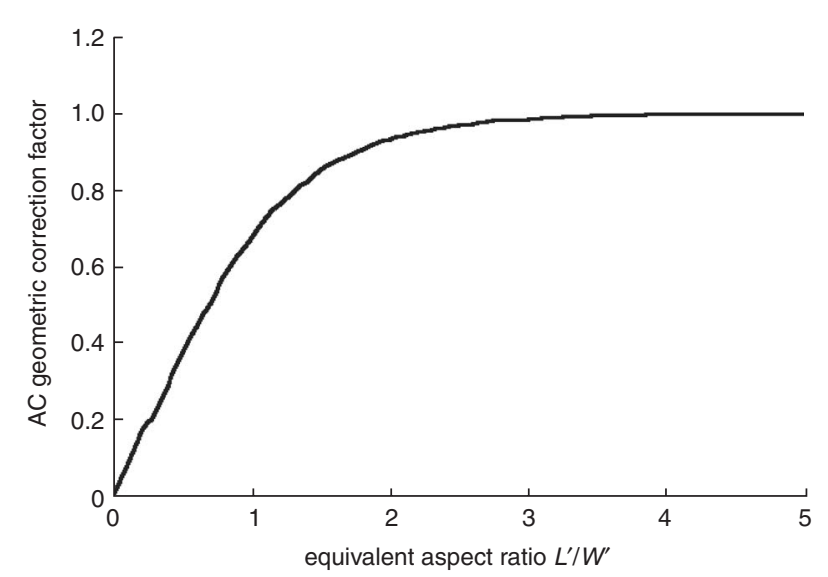

Fig. 5 Simulation results for the geometric correction factor $G_{A C}$ with respect to equivalent aspect ratios $L^{\prime} / W^{\prime}$

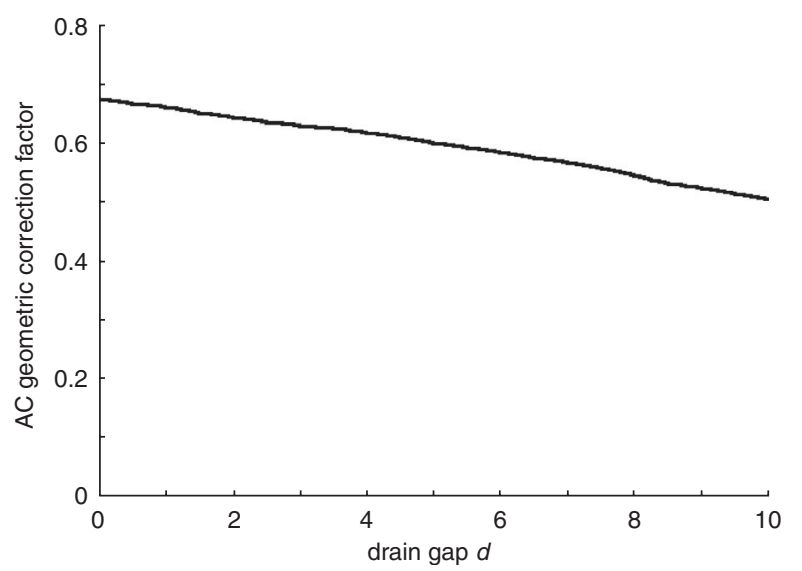

Fig. 6 Simulation results for the geometric correction factor $G_{A C}$ with respect to the drain gap

sensitivity, which is based on an inherent small bias current due to high aspect ratio $\left(L^{\prime} / W^{\prime}\right)$, reduce the performance of this ideal assembly.

\section{Experimental results}

A bias voltage is applied to the gate of the concave or convex MAGFET to yield a hybrid bias voltage $V_{\text {bias }}=3.0 \mathrm{~V}+0.5 \mathrm{~V} \times \sin (2 \pi f t)$. The magnitude of the bias voltage is experimentally measured. A significant readout must be obtained and the two split DC drain currents, $I_{D 1}$ and $I_{D 2}$, must be almost equal. Figure 7 plots the measured DC bias currents, $I_{D 1}$ and $I_{D 2}$, for a concave MAGFET with parameters $L / W=40 \mu \mathrm{m} / 40 \mu \mathrm{m}$ and $d=2 \mu \mathrm{m}$. This Figure shows that the section from $V_{\text {bias }}=2.5 \mathrm{~V}$ to $V_{\text {bias }}=3.5 \mathrm{~V}$, is almost linear and meets the criterion $I_{D 1} \approx I_{D 2}$.

With respect to the frequency modulation, the frequency of AC bias must be sufficiently high, typically above $100 \mathrm{kHz}$ [5]. However, Panina proposed that a low frequency is acceptable [14]. The compromise is to use a modulating frequency of $100 \mathrm{kHz}$. The applied aspect ratios $(L / W)$ are selected as $40 \mu \mathrm{m} / 80 \mu \mathrm{m}, 40 \mu \mathrm{m} / 40 \mu \mathrm{m}$ and $40 \mu \mathrm{m} / 20 \mu \mathrm{m}$, and the drain gaps $(d)$ are $2 \mu \mathrm{m}, 4 \mu \mathrm{m}$ and $6 \mu \mathrm{m}$, respectively. First, the magnetic device, whose drain gap is fixed at $2 \mu \mathrm{m}$, is considered to obtain the optimum geometric parameter. Measurement results indicate that the optimum aspect ratio is $40 \mu \mathrm{m} / 40 \mu \mathrm{m}$. Secondly, the drain gaps are expanded from $2 \mu \mathrm{m}$ to $6 \mu \mathrm{m}$ by fixing the optimum aspect ratio. All the measurements are taken with

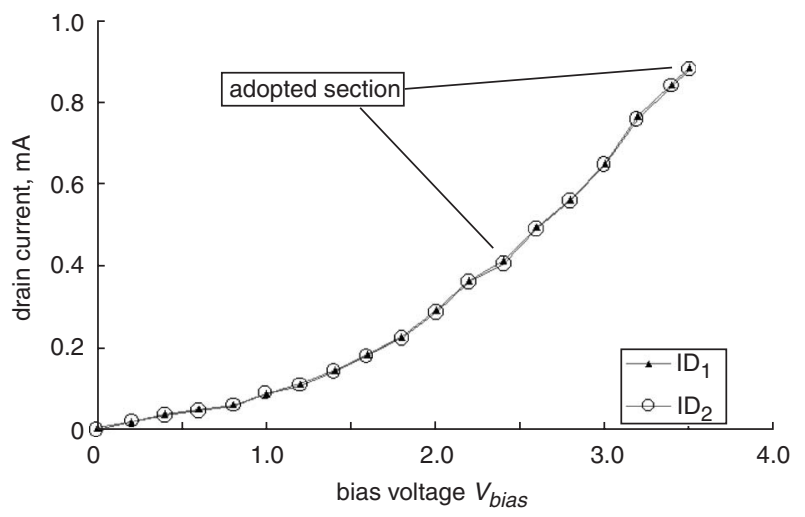

Fig. 7 Measured results for DC bias current $I_{D 1}$ and $I_{D 2}$ for the concave MAGFET $(L / W=40 \mu \mathrm{m} / 40 \mu \mathrm{m}$ and $d=2 \mu \mathrm{m})$

Section from $V_{\text {bias }}=2.5 \mathrm{~V}$ to $V_{\text {bias }}=3.5 \mathrm{~V}$ is almost linear and fits the basic decision criterion $I_{D 1} \approx I_{D 2}$ 
an applied DC magnetic field that varies from $-150 \mathrm{mT}$ to $150 \mathrm{mT}$, and are recorded by a Lockin Voltmeter (NF Electronic Instruments, Model: 5560). Table 1 and 2 show the measured results at a modulating frequency of $f=100 \mathrm{kHz}$ and a DC bias current, respectively. The absolute sensitivity, $S_{A}$, the supply-current-related sensitivity $S_{R I}$ and the supply-voltage-related sensitivity $S_{R V}$ are defined as in [7].

$$
\begin{gathered}
S_{A}=\left|\frac{\Delta V_{\text {out }}}{\Delta B}\right|[\mathrm{V} / \mathrm{T}] \\
S_{R I}=\left|\frac{1}{I_{A C}} \frac{\Delta V_{\text {out }}}{\Delta B}\right|[\mathrm{V} / \mathrm{A} \cdot \mathrm{T}] \\
S_{R V}=\left|\frac{1}{V_{A C}} \frac{\Delta V_{\text {out }}}{\Delta B}\right|[\mathrm{V} / \mathrm{V} \cdot \mathrm{T}]
\end{gathered}
$$

The supply $\mathrm{AC}$ bias current $I_{A C}$ is defined as $I_{A C}=$ $I_{A C 1}+I_{A C 2}$ and the AC bias voltage $V_{A C}$, is fixed at $0.5 \mathrm{~V}$. Figures 8 and 9 present the measured (dots) and simulated (solid line) results of induced output voltages with respect to aspect ratios and drain gaps for concave and convex MAGFETs, respectively.

Furthermore, the geometric correction factor must be modified to compensate for the measured current difference.
Figures 10 and 11 present the measured and simulated results for geometric correction factors as functions of aspect ratios and drain gaps for concave and convex MAGFETs, respectively. The dots in the Figures indicate the measured values defined in (1), and the solid lines are the simulated values calculated by (19) with error correction factors, obtained by computer simulation, and shown in Table 3. Notably, four MAGFETs were placed in combination to enhance the AC output voltage and inhibit the unwanted noise. Figure 12 presents a chip microphotograph of this new design.

\section{Discussion}

Table 1 indicates that the highest values of absolute sensitivity $S_{A}$, supply-current-related sensitivity $S_{R I}$ and supply-voltage-related sensitivity $S_{R V}$, are $88.82 \mathrm{mV} / \mathrm{T}$, $2121.74 \mathrm{~V} / \mathrm{A}$.T and $177.65 \mathrm{mV} / \mathrm{V}$.T for the concave MAGFET, and $261.34 \mathrm{mV} / \mathrm{T}, 6008.26 \mathrm{~V} / \mathrm{A} . \mathrm{T}$ and $522.68 \mathrm{mV} / \mathrm{V} . \mathrm{T}$ for the convex MAGFET with a $0.5 \mathrm{~V}$ AC bias, respectively. These results show that the sensitivities of a convex MAGFET are always greater than those of a concave MAGFET, and that the optimum design is that of the convex MAGFET built with the geometric parameters $L / W=40 \mu \mathrm{m} / 40 \mu \mathrm{m}$ and $d=2 \mu \mathrm{m}$. Despite these favourable

Table 1: Summary of geometric parameters $L / W$ and $d, A C$ bias current $I_{A C}$, absolute sensitivity $S_{A}$, supply-current-related

\begin{tabular}{|c|c|c|c|c|c|c|}
\hline Category & $L / W(\mu \mathrm{m} / \mu \mathrm{m})$ & $d(\mu \mathrm{m})$ & $I_{\mathrm{AC}}(\mathrm{mA})$ & $S_{\mathrm{A}}(\mathrm{mV} / \mathrm{T})$ & $S_{\mathrm{RI}}(\mathrm{V} / \mathrm{A} . \mathrm{T})$ & $S_{\mathrm{RV}}(\mathrm{mV} / \mathrm{N} . \mathrm{T})$ \\
\hline \multirow[t]{5}{*}{ Concave MAGFET } & $40 / 20$ & 2 & 0.0023 & 4.880 & 2121.739 & 9.760 \\
\hline & $40 / 40$ & 2 & 0.0464 & 88.824 & 1914.310 & 177.648 \\
\hline & $40 / 80$ & 2 & 0.1342 & 19.112 & 142.414 & 38.224 \\
\hline & $40 / 40$ & 4 & 0.0777 & 35.776 & 460.438 & 71.552 \\
\hline & $40 / 40$ & 6 & 0.0466 & 14.224 & 305.236 & 28.448 \\
\hline \multirow[t]{5}{*}{ Convex MAGFET } & $40 / 20$ & 2 & 0.0385 & 233.628 & 6008.260 & 467.256 \\
\hline & $40 / 40$ & 2 & 0.0557 & 261.340 & 4691.921 & 522.680 \\
\hline & $40 / 80$ & 2 & 0.1218 & 89.228 & 732.578 & 178.456 \\
\hline & $40 / 40$ & 4 & 0.0557 & 21.624 & 388.223 & 43.248 \\
\hline & $40 / 40$ & 6 & 0.0699 & 20.108 & 287.668 & 40.216 \\
\hline
\end{tabular}
sensitivity $S_{R I}$ and supply-voltage-related sensitivity $S_{R V}$ for concave and convex MAGFETs with a modulating frequency of $f=100 \mathrm{kHz}$

\begin{tabular}{|c|c|c|c|c|c|c|}
\hline Category & $L / W(\mu \mathrm{m} / \mu \mathrm{m})$ & $d(\mu \mathrm{m})$ & $I_{\mathrm{AC}}(\mathrm{mA})$ & $S_{\mathrm{A}}(\mathrm{mV} / \mathrm{T})$ & $S_{\mathrm{RI}}(\mathrm{V} / \mathrm{A} . \mathrm{T})$ & $S_{\mathrm{RV}}(\mathrm{mV} / \mathrm{N} . \mathrm{T})$ \\
\hline \multirow[t]{5}{*}{ Concave MAGFET } & $40 / 20$ & 2 & 0.026 & 70.0 & 2004.8 & 34.8 \\
\hline & $40 / 40$ & 2 & 0.475 & 1122.0 & 1464.6 & 463.8 \\
\hline & $40 / 80$ & 2 & 3.107 & 18.0 & 3.4 & 6.9 \\
\hline & $40 / 40$ & 4 & 1.172 & 59.0 & 25.6 & 20.0 \\
\hline & $40 / 40$ & 6 & 0.962 & 19.0 & 9.0 & 5.8 \\
\hline \multirow[t]{5}{*}{ Convex MAGFET } & $40 / 20$ & 2 & 0.773 & 46.0 & 77.2 & 39.8 \\
\hline & $40 / 40$ & 2 & 0.858 & 433.0 & 512.4 & 292.9 \\
\hline & $40 / 80$ & 2 & 1.642 & 112.0 & 117.6 & 128.7 \\
\hline & $40 / 40$ & 4 & 0.871 & 90.0 & 56.0 & 32.5 \\
\hline & $40 / 40$ & 6 & 0.902 & 11.0 & 3.8 & 2.3 \\
\hline
\end{tabular}

Table 2: Summary of geometric parameters $L / W$ and $d, D C$ bias current $I_{D C}$, absolute sensitivity $S_{A}$, supply-current-related sensitivity $S_{R l}$, and supply-voltage-related sensitivity $S_{R V}$ for concave and convex MAGFETs with DC bias current 


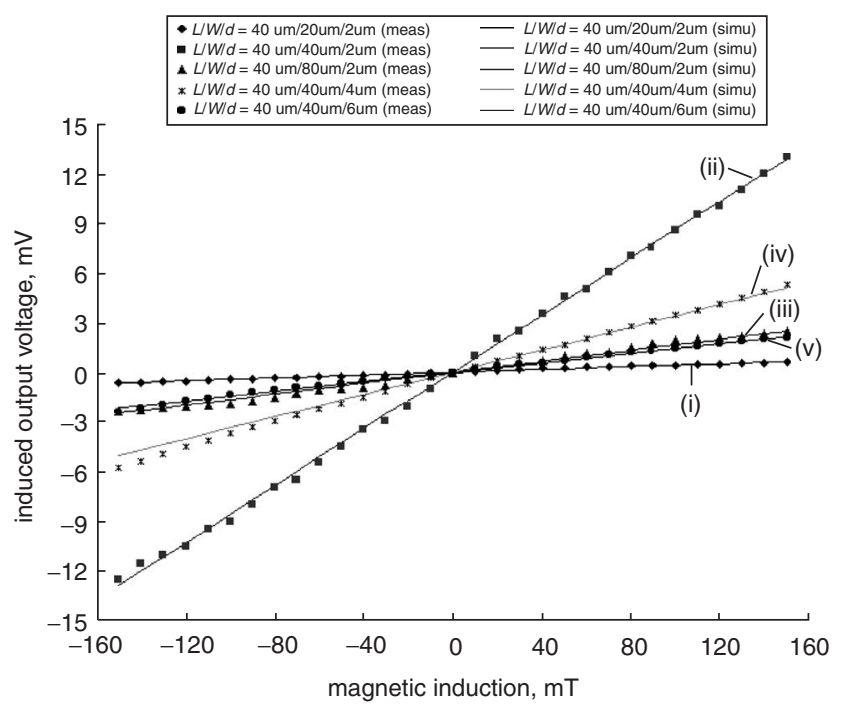

Fig. 8 Measured and simulated results for induced AC output voltages for the concave MAGFET

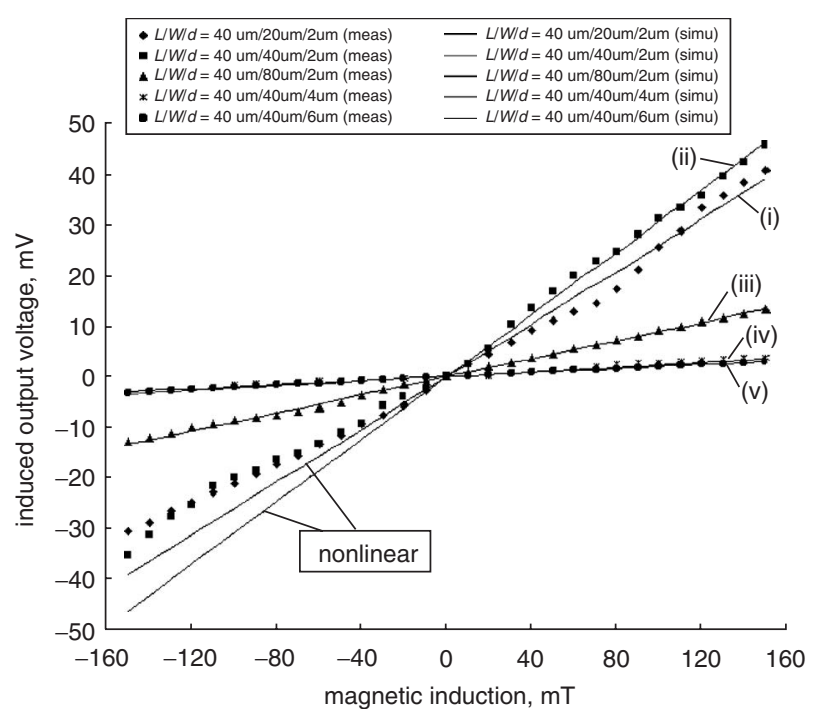

Fig. 9 Measured and simulated results for induced AC output voltages for the convex MAGFET

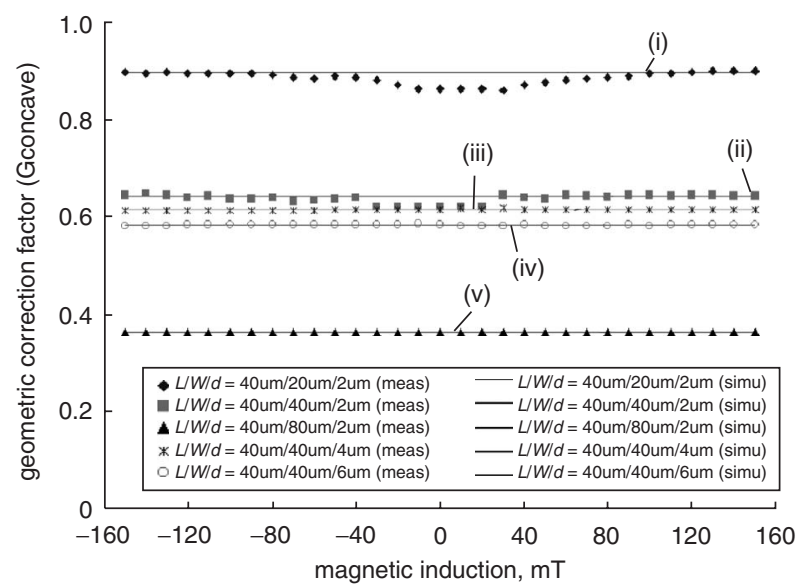

Fig. 10 Measured and simulated results for geometric correction factors with respect to aspect ratios and drain gaps for the concave MAGFET

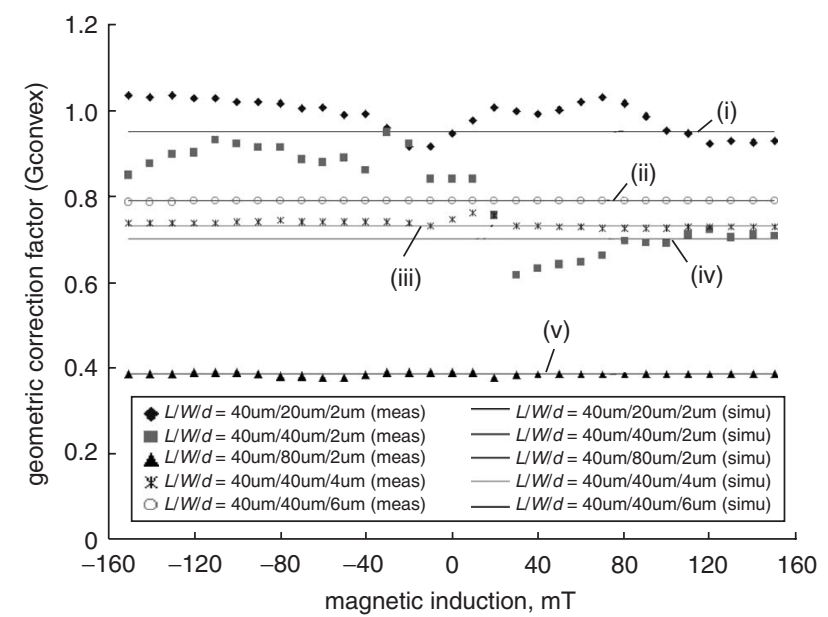

Fig. 11 Measured and simulated results for geometric correction factors with respect to aspect ratios and drain gaps for the convex MAGFET

Table 3: Summary of the simulated error correction factors $E_{\text {concave }}$ and $E_{\text {convex }}$ for concave and convex MAGFETs with a modulating frequency of $f=100 \mathrm{kHz}$

\begin{tabular}{lllll}
\hline Category & $\begin{array}{l}L W \\
(\mu \mathrm{m} / \mu \mathrm{m})\end{array}$ & $d(\mu \mathrm{m})$ & $K_{\text {concave }}$ & $K_{\text {convex }}$ \\
\hline Concave MAGFET & $40 / 20$ & 2 & -1.980 & - \\
& $40 / 40$ & 2 & -2.520 & - \\
& $40 / 80$ & 2 & -5.190 & - \\
& $40 / 40$ & 4 & -3.005 & - \\
& $40 / 40$ & 6 & -3.215 & - \\
Convex MAGFET & $40 / 20$ & 2 & - & -1.440 \\
& $40 / 40$ & 2 & - & -1.442 \\
& $40 / 80$ & 2 & - & -4.371 \\
& $40 / 40$ & 4 & - & -2.540 \\
& $40 / 40$ & 6 & - & -2.388 \\
\hline
\end{tabular}

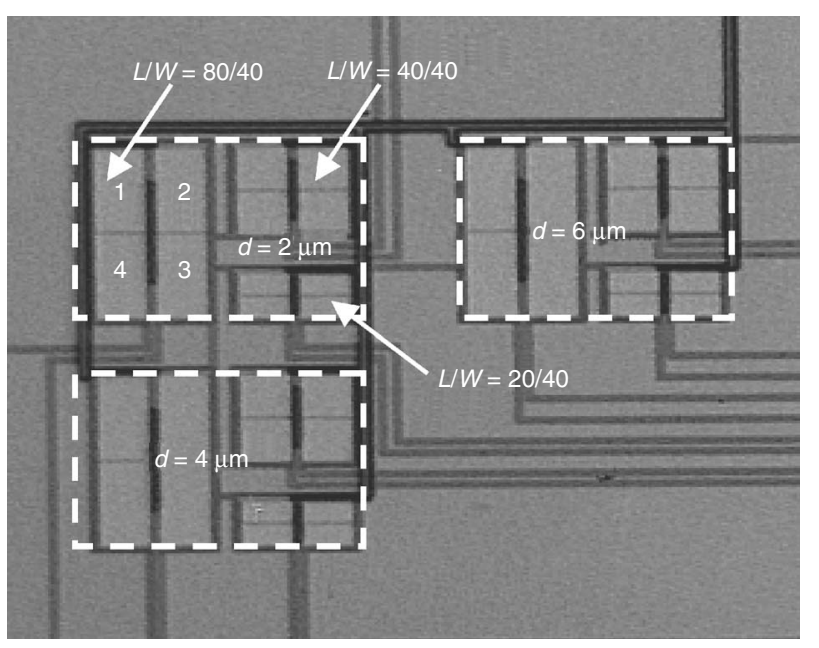

Fig. 12 Chip microphotograph for an array (four sets) of concave MAGFETS

properties, the device fabricated with a high aspect ratio and a low drain gap is not the best assembly for a linear output because of its nonlinearity.

Table 2 indicates that the highest values of absolute sensitivity $S_{A}$, supply-current-related sensitivity $S_{R I}$ and

IEE Proc.-Circuits Devices Syst., Vol. 151, No. 6, December 2004 
supply-voltage-related sensitivity $S_{R V}$ are $1122.0 \mathrm{mV} / \mathrm{T}$, 2004.8 V/A.T and $463.8 \mathrm{mV} / \mathrm{V}$.T for the concave MAGFET, and $433.0 \mathrm{mV} / \mathrm{T}, 512.4 \mathrm{~V} / \mathrm{A} . \mathrm{T}$ and $292.9 \mathrm{mV} / \mathrm{V} . \mathrm{T}$ for the convex MAGFET with the DC bias current, respectively. These results show that the maximum sensitivities of a concave MAGFET are always greater than those of a convex MAGFET, and that the optimum design is that of the concave MAGFET built with the geometric parameters $L / W=40 \mu \mathrm{m} / 40 \mu \mathrm{m}$ and $d=2 \mu \mathrm{m}$. Comparing these results with Table 1 , the optimum design is the concave MAGFET for DC bias, but the convex MAGFET for AC bias.

It should be noted, however, enlarging the aspect ratio $(L / W)$ cannot effectively increase the sensitivity of a MAGFET with AC bias, the evidence for which is very different from that for a conventional MAGFET. Although reducing the aspect ratio $(L / W)$ increases the $\mathrm{AC}$ bias current (as shown in Table 1), the enhancing effect of a large AC bias current is cancelled out by a huge increment in the amplitude of the error correction factor. In general, a lower aspect ratio corresponds to a higher amplitude of the error correction factor. Furthermore, increasing the drain gap reduces the sensitivity more slowly than minimising the aspect ratio. Nevertheless, reducing the aspect ratio and broadening the drain gap can improve the linear characteristics of both concave and convex MAGFETs. Figures 8 and 9 plot the measured output voltages of concave and convex MAGFETs, respectively. Although the sensitivity of the convex MAGFET is typically greater than that of the concave MAGFET, the nonlinearity seriously inhibits the functioning of the convex MAGFET manufactured with a high aspect ratio. A concave MAGFET is a very good device for industrial applications because of its fine linear output; however, the convex MAGFET is another good choice in switch design, because of its high sensitivity.

Figures 10 and 11 show the measured and simulated geometric correction factors for concave and convex MAGFETs, respectively. The measurement results closely correspond to the simulated results, but curves (i) and (ii) include some errors at low magnetic field. Even so, the transformed model is suited to a magnetic device with a low aspect ratio and a high drain gap. Unfortunately, the compromise is low sensitivity. The convex MAGFET has the same shortcoming. In Fig. 11, the measured results for curves (i) and (iv) are too poor to be acceptable. Notably, the sensitivity in the narrow drain gap exceeds that in the broad drain gap, but the nonlinearity makes this advantage completely worthless. The best solution is to magnify the drain gap, as proven by curves (ii) and (iii) in Fig. 11. Given a consistent geometric correction factor (curves (iii), (iv) and (v) in Fig. 10, and curves (ii), (iii) and (v) in Fig. 11), the concave MAGFET with geometric parameters $L / W=40 \mu \mathrm{m} / 40 \mu \mathrm{m}$ and $d=4 \mu \mathrm{m}$ is the best choice and the convex MAGFET with geometric parameters $L / W=40 \mu \mathrm{m} / 80 \mu \mathrm{m}$ and $d=2 \mu \mathrm{m}$ is also excellent. In a concave MAGFET, the well situated drain gap $(d=4 \mu \mathrm{m})$ easily separates the split drain current and yields a significant current difference when a DC magnetic induction is applied. However, a parasitic MOSFET is contained by split drains of the convex MAGFET, narrowing the well situated drain gap to $2 \mu \mathrm{m}$.

\section{Conclusions}

This study has theoretically derived geometric correction factors with error correction factors for both concave and convex split-drain MAGFETs. All geometric correction factors were empirically verified by measurement. For the concave MAGFET, the highest absolute sensitivity $S_{A}$, the supply-current-related sensitivity $S_{R I}$ and the supply-voltage-related sensitivity $S_{R V}$ are $88.82 \mathrm{mV} / \mathrm{T}$, $2121.74 \mathrm{~V} / \mathrm{A} . \mathrm{T}$ and $177.65 \mathrm{mV} / \mathrm{V} . \mathrm{T}$ at a $0.5 \mathrm{~V}$ AC bias voltage, respectively. Also, prime sensitivities $S_{A}=$ $261.34 \mathrm{mV} / \mathrm{T}, S_{R I}=6008.26 \mathrm{~V} / \mathrm{A} . \mathrm{T}$ and $S_{R I}=522.68 \mathrm{mV} /$ V.T are obtained under the same bias conditions as for the convex MAGFET. Expanding the drain gap and minimising the aspect ratio reduce the nonlinearity of both concave and convex MAGFETs, but at the cost of reducing magnetosensitivities.

The concave MAGFET is the best choice for linear output because of its linearity, but the convex MAGFET is another prime choice for switch design due to its high sensitivity. Despite the fact that the sensitivity of the narrow drain gap exceeds that of the broad drain gap, the nonlinearity seriously restricts the convex MAGFET's application. If the consistent geometric correction factor is emphasised in the theoretical derivation, then the convex MAGFET with geometric parameters, $L / W=40 \mu \mathrm{m} / 80 \mu \mathrm{m}$ and $d=2 \mu \mathrm{m}$, and the concave MAGFET with geometric parameters, $L / W=40 \mu \mathrm{m} / 40 \mu \mathrm{m}$ and $d=4 \mu \mathrm{m}$ are preferred. Besides, the optimum design is the concave MAGFET for DC bias, but that of the convex MAGFET for AC bias.

\section{Acknowledgments}

The authors would like to thank the National Science Council of the Republic of China, Taiwan for financially supporting this research under contract NSC 90-2218-E027-020. The authors thank CIC, Taiwan for fabricating the MAGFET test chips.

\section{References}

1 Popovic, R.S., and Baltes, H.P.: 'A MOS magnetic field sensor', IEEE J. Solid-State Circuits, 1983, 18, pp. $426-428$

2 Yang, H.M., and Lei, T.F.: 'The DC circuit model of a MOS magnetic sensor', Sens. Actuators A, Phys., 1997, 58, pp. 125-127

3 Nathan, A., Andor, L., Baltes, H.P., and Weinmar, H.G.: 'Modeling of a dual-drain NMOS magnetic-field sensor', IEEE J. Solid-State Circuits, 1985, 20, (3), pp. 819-821

4 Killat, D., Kluge, J.V., Umbach, F., Langheinrich, W., and Schmitz, R.: 'Measurement and modeling of sensitivity and noise of MOS magnetic field-effect transistors', Sens. Actuators A, Phys., 1997, 61, pp. $346-351$

5 Aragoneses, P., Zhukov, A.P., Gonzalez, J., Blanco, J.M., and Dominguez, L.:' 'Effect of AC driving current on magneto-impedance effect', Sens. Actuators A, Phys., 2000, 81, pp. 86-90

6 Johannes, W.A., and Langheinrich, W.A.: 'An analytical model of MAGFET sensitivity including secondary effects using a continuous description of the geometric correction factor G', IEEE Trans. Electron Devices, 1999, 46, pp. 89-95

7 Popovic, R.S.: 'Hall-effect devices', Sens. Actuators A, Phys., 1989, 17, pp. 39-53

8 Liu, S.I., Wei, J.F., and Sung, G.M.: 'SPICE macro model of MAGFET and its applications', IEEE Trans. Circuits Syst. II, Analog Digit. Signal Process., 1999, 46, (4), pp. 370-375

9 Roumenin, C.S.: 'Handbook of sensors and actuators: solid state magnetic sensors' (Elsevier, Amsterdam, The Netherlands, 1994)

10 Trujillo, H., Nagy, A., and Cruz, J.C.: 'Influence of topology on the response of lateral magnetotransistors', Sens. Actuators A, Phys., 1994, 45, pp. $179-182$

11 Clark, J.J.: 'Split-drain MOSFET magnetic sensor arrays', Sens. Actuators A, Phys. 1990, 24, pp. 107-116

12 Nathan, A., Huzser, A., and Baltes, H.: 'Two-dimensional numerical modeling of magnetic-field sensors in CMOS technology', IEEE Trans. Electron Devices, 1985, 32, pp. 1212-1219

13 Baltes, H.P., and Popovic, R.S.: 'Integrated semiconductor magnetic field sensors', Proc. IEEE, 1986, 74, (8), pp. 1107-1132

14 Sze, S.M.: 'Physics of semiconductor devices' (Bell Lab., Inc., Murray Hill, NJ, USA, 1985, 2nd edn.)

15 O'Neil, P.V.: 'Advanced engineering mathematics' (Wadsworth Publishing Company, Belmont, CA, USA, 1987) 


\section{Appendix: Voltage distribution $V(x, z)$ in equivalent rectangle for $A C$ bias}

According to the method of the separation of variables (Fourier method) and the superposition method [15], two cases must be considered.

Case 1: $E_{x}(x, 0)=E_{x}\left(x, L^{\prime}\right)=E_{x}(0, z)=0$ and $E_{x}\left(W^{\prime}, z\right)$ $=E_{H A C}$.

The separation of variables method yields a solution with the following form:

$$
V(x, z)=X(x) \cdot Z(z)
$$

where $X(x)$ is the voltage distribution in the $x$-direction and $Z(z)$ is the voltage distribution in the $z$-direction. Including initial and boundary conditions, the voltage distribution $V$ $(x, z)$ is

$$
V(x, z)=\sum_{n=1}^{\infty} A_{n} \cosh \left(\frac{n \pi}{L^{\prime}} x\right) \sin \left(\frac{n \pi}{L^{\prime}} z\right)
$$

and

$$
\begin{aligned}
E_{x}(x, z)= & -\frac{d[V(x, z)]}{d x} \\
& =-\sum_{n=1}^{\infty} A_{n} \cdot \frac{n \pi}{L^{\prime}} \cdot \sinh \left(\frac{n \pi}{L^{\prime}} x\right) \cdot \sin \left(\frac{n \pi}{L^{\prime}} z\right)
\end{aligned}
$$

To satisfy $E_{x}\left(W^{\prime}, z\right)=E_{H A C}$, try a superposition

$$
E_{H A C}=\sum_{n=1}^{\infty}-A_{n} \cdot \frac{n \pi}{L^{\prime}} \cdot \sinh \left(\frac{n \pi}{L^{\prime}} W^{\prime}\right) \cdot \sin \left(\frac{n \pi}{L^{\prime}} z\right)
$$

The constant $A_{n}$ is then calculated as

$$
A_{n}=\frac{2 E_{H A C} \cdot L^{\prime} \cdot\left[(-1)^{n}-1\right]}{(n \pi)^{2}} \cdot \frac{1}{\sinh \frac{n \pi}{L^{\prime}} W^{\prime}}
$$

Hence, the solution of case 1 is

$$
\begin{aligned}
V(x, z)= & -\int_{0}^{W^{\prime}} E_{x}(x, z) d x+f_{1}(z) \\
= & \sum_{n=1}^{\infty}\left[\frac{2 E_{H A C} \cdot L^{\prime} \cdot\left[(-1)^{n}-1\right]}{(n \pi)^{2}} \cdot \frac{1}{\sinh \frac{n \pi}{L^{\prime}} W^{\prime}}\right] \\
& \times\left[\cosh \frac{n \pi}{L^{\prime}} x \cdot \sin \frac{n \pi}{L^{\prime}} z\right]+f_{1}(z)
\end{aligned}
$$

where $f_{1}(z)$ is the voltage distribution not in terms of the variable $x$.
Case 2: $E_{x}(x, 0)=E_{x}\left(x, L^{\prime}\right)=E_{x}\left(W^{\prime}, z\right)=0$ and $E_{x}(0, z)$ $=E_{H A C}$

As in case 1, the electric field $E_{x}(x, z)$ and the constant $B_{n}$ are given by

$$
\begin{aligned}
& E_{x}(x, z) \\
& =-\sum_{n=1}^{\infty}\left(B_{n} \cdot \frac{n \pi}{L^{\prime}}\right)\left(\sinh \frac{n \pi}{L^{\prime}} x-\tanh \frac{n \pi}{L^{\prime}} W^{\prime} \cdot \cosh \frac{n \pi}{L^{\prime}} x\right) \\
& \cdot \sin \left(\frac{n \pi}{L^{\prime}} z\right)
\end{aligned}
$$

and

$$
B_{n}=-\frac{2 L^{\prime} \cdot E_{H A C} \cdot\left[(-1)^{n}-1\right]}{(n \pi)^{2}} \cdot \frac{1}{\tanh \frac{n \pi}{L^{\prime}} W^{\prime}}
$$

Hence, the solution of case 2 is

$$
\begin{aligned}
V(x, z)= & \sum_{n=1}^{\infty}\left[\frac{-2 L^{\prime} \cdot E_{H A C} \cdot\left[(-1)^{n}-1\right]}{(n \pi)^{2}} \cdot \frac{1}{\tanh \frac{n \pi}{L^{\prime}} W^{\prime}}\right] \\
& \times\left[\cosh \frac{n \pi}{L^{\prime}} x-\tanh \frac{n \pi}{L^{\prime}} W^{\prime} \cdot \sinh \frac{n \pi}{L^{\prime}} x\right] \\
& \cdot \sin \frac{n \pi}{L^{\prime}} z+f_{2}(z)
\end{aligned}
$$

where $f_{2}(z)$ is the voltage distribution not in terms of the variable $x$.

Combining (24) and (25)

$$
\begin{aligned}
V(x, z)= & \sum_{n=1}^{\infty}\left[\frac{2 L^{\prime} \cdot E_{H A C} \cdot\left[(-1)^{n}-1\right]}{(n \pi)^{2}} \cdot \frac{1}{\sinh \frac{n \pi}{L^{\prime}} W}\right] \\
& \cdot\left[\cosh \frac{n \pi}{L^{\prime}} x \cdot \sin \frac{n \pi}{L^{\prime}} z\right] \\
& +\sum_{n=1}^{\infty}\left[\frac{-2 L^{\prime} \cdot E_{H A C} \cdot\left[(-1)^{n}-1\right]}{(n \pi)^{2}} \cdot \frac{1}{\tanh \frac{n \pi}{L^{\prime}} W}\right] \\
& \cdot\left(\cosh \frac{n \pi}{L^{\prime}} W \cdot \tanh \frac{n \pi}{L^{\prime}} W \cdot \sinh \frac{n \pi}{L^{\prime}} x\right) \cdot \sin \frac{n \pi}{L^{\prime}} z \\
& +f(z)
\end{aligned}
$$

where the function $f(z)$ is the voltage distribution in the $z$-direction and equals the sum of $f_{1}(z)$ and $f_{2}(z)$. 